\title{
Research on the Photocatalytic-oxidating Degradation of Organic Dyes Lin Chen ${ }^{1, a}$ \\ ${ }^{1}$ School of Chemical Engineering, Liaoning University of Science and Technology, Anshan, Liaoning, 114044 \\ ${ }^{a}$ email
}

Keywords: Photocatalytic-oxidating Degradation, Organic Dyes

\begin{abstract}
Treatment of toxic organic wastewater biorefractory due to lack of effective treatment methods became the difficulties in the field of water, humans and the environment caused great harm. In this paper, lead dioxide doped vanadium and titanium thin films were characterized and Photocatalytic. On this basis, the preparation of lead dioxide electrodes have a higher oxygen evolution potential and a certain catalytic activity, and the characterization and study of Visible Light Degradation of $\mathrm{RhB}$ proposed photoelectric degradation mechanisms under visible light irradiation.
\end{abstract}

\section{Introduction}

Since the industrial revolution, scientific and technological progress of mankind in the economic development and the number on the scale has made remarkable achievements. However, excessive use of resources and energy, a lot of pollutant emissions, large-scale human-caused environmental disasters continue to occur, and some has become a global problem threatening human survival and development, and therefore, the effective use of resources and protection of the environment and degradation repair the environment is today's most important issue. Water and air pollution is an important indicator of environmental degradation. Organic pollution is the most common aqueous environment pollution, the United States announced 129 EPA priority pollutants, Germany announced 120 EPA priority pollutants, our published 58 kinds, such as methylene chloride, chloroform, tetrachloromethane methane, chlorobenzene, phenol, aniline, which is the most toxic compounds containing chlorine and nitrogen, for many having a benzene ring structure. These contaminants or directly to the source of sewage or sewage organic degradation products, which are widely distributed in soil, surface water, groundwater, sediment and water, contaminating the food chain. The current sewage treatment methods are: sedimentation, flocculation, filtration, adsorption, flotation, biological treatment, but these methods are inevitably bring secondary pollution, and expensive regeneration or long processing cycle. Although sewage by conventional biological treatment effective treatment, but there are still hundreds of highly toxic compounds can not be treated with biological. Especially high concentration of highly toxic Governance biorefractory organic wastewater over a longer period of time will become difficult and the focus of environmental governance, cost-effective easy to explore new technology industrialization process is very important. Currently treatment of organic wastewater advanced oxidation technologies include: chemical oxidation, wet air oxidation, supercritical water oxidation, chemical oxidation acoustic irradiation oxidation, pulse corona method, photocatalysis, electrochemical and catalytic each method the catalytic process.

\section{Photocatalytic Technology}

The initial photocatalytic reaction is initiated which catalyzes the reaction of light, where the photocatalyst is actually photochemical sensitizers. Modern photocatalyst is not only the so-called light photochemical sensitizers considerable substance. Photocatalytic reaction between the closely linked photochemical and catalytic reactions. Currently photocatalysis is strict definition is still relatively difficult, especially in many cases, the reaction mechanism is uncertain. For the photocatalytic reaction studies must also adopt research methods and theories in order to obtain 
multi-disciplinary. Usually heterogeneous photocatalysis can be considered: adsorption by the photoexcited semiconductor and photochemical reactions in the gas or liquid substance or a solid stretch interface solid-liquid interface.

\section{The Photocatalytic Treatment of Organic Contaminants}

EPA published 129 kinds of basic pollutants, there are nine categories 114 kinds of organic compounds. Thus, photocatalytic wastewater treatment technology focused on the photocatalytic oxidation of organic pollutants in this direction, especially photocatalytic technology can completely destroy a variety of organic compounds, oxidation and decomposition of $\mathrm{COZ}, \mathrm{H} 20$, and other inorganic components such as $\mathrm{Hel}, \mathrm{HBr}$ and the like.

The study found that a large number of wastewater treatment technology with Tio2 photocatalyst containing organic contaminants in water halogenated aliphatic hydrocarbons, halogenated aromatic hydrocarbons, organic acids, dyes, aromatic nitro group, a substituted aniline, polycyclic aromatic hydrocarbons, heterocyclic compounds, hydrocarbons, phenols, surfactants, pesticides, etc. can be effectively treated photocatalytic reaction in addition to drugs, bleaching, mineralization, and ultimately broken down into $\mathrm{COZ}$ and $\mathrm{HZO}$, which eliminated the pollution of the environment. However, most of the research is carried out in a suspension system, using powdered photocatalyst. However, the presence of the photocatalyst powder separation and recovery difficult problem during use, in fact, this has become the photocatalytic titanium dioxide method based on one of the main commercial difficult. Feasibility Therefore, since the late 1990s, the United States, Japan and other countries to carry out the preparation of immobilized titanium dioxide film (coating) research and titanium dioxide powder, so far, it has experimented with a variety of membrane preparation techniques .

Depth treatment of drinking water, the traditional drinking water treatment process can not effectively remove trace organic pollutants in water and chlorination process has produced a number of halogenated organic compounds, which are mostly carcinogenic, teratogenic, mutagenic three-induced substance. However, with the TIO: photocatalytic treatment of drinking water, which can effectively remove the above hazardous substances.

With interior decoration materials, it uses household chemicals, indoor air pollution, more and more people's attention. Indoor used in coatings, adhesives, paints, glue boards will release volatile organic compounds into the air dryer caused by indoor air pollution, indoor air from the currently detected hundreds of organic compounds, some of which are carcinogens. The findings show that some organic compounds in indoor air concentrations higher than outdoors, even higher than the industrial zone. Photocatalytic air purifier germicidal lamp is generally used in a medical source as a reaction can simultaneously achieve indoor air purification and air to kill pathogenic microorganisms purpose

At home and abroad has been olefins, alcohols, ketones, aldehydes, aromatic compounds, organic acids, organic compounds, trichlorethylene and other organic compounds in gaseous photocatalytic degradation study, results showed that the photocatalytic activity of the gas phase of organic pollutants are generally more aqueous the much higher, which is due to the high mass transfer rate of molecular diffusion in the gas phase and the chain reaction easier. Studies have shown that use of a photocatalyst to TIOZ as the main component can be successfully removed 1-260mg.m-3 of acetone, butanol, butyraldehyde, formaldehyde and m-xylene and other organic pollutants, and formaldehyde and toluene is precisely the interior the existence of major air pollutants.

\section{Electrochemical Catalytic Technology}

Electrochemical Catalysis by electrode reaction of ozone or strong oxidants such as hydrogen peroxide, and converted to a more appropriate by the radicals under controlled conditions, so as to effectively degrade refractory organics, also known as electro-catalytic oxidation of organic compounds . For a long time, limited by the electrode material, degradation of organic matter 
oxidation catalyst electric current inefficient, high power consumption, it is difficult to achieve industrialization. 80 years later, many researchers from home and abroad to develop electro-catalytic active electrode material proceed on organic electro-catalytic oxidation mechanism and influencing factors were studied, achieved a major breakthrough, and began to be used biodegradation of organic wastewater treatment. China also carried out research in this field.

Electrochemical catalysis technology mainly has the following characteristics: (1) in the oxidation process does not have to add any substance; (2) also has the role of disinfection; (3) high energy efficiency, low temperature can be conducted; (4) the device is relatively simple, low operating costs, easy automatic control; (5) no secondary pollution, known as "environmentally friendly processing technology."

\section{The Problems of Electrochemical Catalysis}

Electrochemical catalytic technology as a new type of environmental pollutant reduction technologies, has attracted the attention of researchers States. Number of research institutions at home and abroad have been carrying out many innovative research work and made a lot of achievements. But in general, this technology is still in the development stage at home and abroad, in the degradation mechanism from the excellent level of treatment effectiveness study macroscopic to microscopic scale analyzes are still rudimentary. Based on the above analysis of the literature, the technology also the following deficiencies:

Most of the studies limited to laboratory studies used small electrode area $(1-10 \mathrm{~cm} 2)$, and the actual application of wastewater treatment is still a large distance. So how to conduct experimental basic research and engineering application of organic combination is an inevitable trend to continue to develop the technology. .

Catalytic electrode material impact on the degradation effect is obvious, but its lack of theoretical guidance to develop, if the development of good catalytic performance, high stability at a reasonable cost using electrodes, electrochemical techniques will be able to promote the environmental treatment of organic pollutants revolution development.

In the electrochemical method aspects of organic pollutants, electrochemical reactor and selection of superior grade did not cause enough attention, such as the existing so-called bipolar packed heavy macroscopic treatment effect, while a specific reactor structure, power supply, etc., lack of systematic research.

In terms of degradation mechanism for the direct identification of the reaction mechanism of the lack of active species may be present in the reaction pathway still remain in the speculative stage envisaged, general degradation of certain pollutants no special induction system. In dynamics, research relatively shallow, much less kinetic model than other advanced oxidation processes. And rarely COD or TOC comprehensive index for the object, both of which will be in the actual wastewater treatment is more effective.

Woe electrochemical and other technologies in combination showed good prospects, the current combination of superior grade range still expanding, such as joint research and other advanced oxidation processes belong to the same process of less depth. By examining the study of treatment effect and mechanism of disaster together, eventually forming a composite of several effective electrochemical advanced oxidation technology, it will have a broader application prospects. .

\section{Photoelectrocatalytic}

To further improve the efficiency of the degradation of organic matter, the photochemical catalysis (oxidation) and electrochemical catalytic processes combine to achieve a hot electrocatalytic and photocatalytic technology research on photovoltaic technology synergies of combining technology recently became degraded. Comprehensive literature, such techniques can be roughly divided into two categories. One is in front of the "electric Fenton" process on the basis, supplemented by ultraviolet radiation, thereby forming a "photoelectric Fenton." Brillas leading Spanish research team conducted a deep study of the process. They Pt as anode and a carbon cathode oxygenation 
PTFE cathode and ferrous ions in the solution was added to make the product and cathodic reduction of hydrogen peroxide to form Fenton reaction. The main effect is further introduced UV photolysis complexed iron ions and organic products, thereby contributing to the degradation of organic matter.

\section{The Current Problems}

Despite the heterogeneous photocatalysis has many obvious advantages, applications in organic waste treatment is very attractive, but is not currently fully industrialized, mainly the following problems:

Currently photocatalytic efficiency is not high, very high apparent quantum yield of only a few thousandths of a few percent to the semiconductor carrier recombination rate, the photocatalytic reaction rate is low obstacle photocatalytic wastewater treatment technology industrialization of the main reasons. According Hoffniamn immobilized Tio2 film and other reports, $20 \mathrm{w}$ black light, at a $\mathrm{pH}$ of 3.6 times the catalytic reaction of phenol degradation rate constant $\mathrm{k}$ is $105.40 \mathrm{~mol}$.L-1.min-1. Photocatalytic Degradation of Benzene reported initial reaction rate Yinn Zhang et only o.25mg.L-1.min-1. Difficult engineering applications.

$\mathrm{TiO} 2$ photocatalyst immobilized and contradictions between the photocatalytic efficiency The use of the suspension system, can be more effectively degrade organic matter recovered photocatalyst is a particularly difficult problem to solve; if fixed method, since the photocatalyst surface area decreases, Photocatalytic efficiency also decreases. Thus there is a contradiction between the immobilized photocatalyst and photocatalytic efficiency.

\section{The Method to Solve this Problem}

For the current problems are generally solved in two ways: First, the photocatalyst is fixed; the second is the photocatalytic efficiency. Photocatalytic efficiency in resolving the problem, two-pronged approach, one internal, namely the photocatalyst itself solve the problems; the second is external, using Photoelectrocatalytic degradation of organic matter and organic matter degradation kinetics of the process. This paper will focus on research catalyst and degradation of organic matter dynamics. Trying to solve the photocatalytic efficiency is not high and the light photocatalytic activity of the immobilized catalyst and the problems.

Currently, there are many on $\mathrm{TiO} 2$ photocatalytic, purification, research air handling, water treatment and other aspects, it is becoming a hot topic. However, most of these studies are carried out in a suspension system, using powdered photocatalyst. In order to address the presence of powdery catalyst separation and recovery difficult, easy to unite, not suitable flow system and other shortcomings, so that as soon as possible commercialization. More effective method is to load photocatalyst prepared. Therefore, since the late 1990s, the United States, Japan and other countries to carry out research immobilized titanium dioxide or titanium dioxide thin films prepared

Titanium dioxide, titanium dioxide membrane preparation is the precursor coated on the substrate surface of the substrate so that the film-forming layer of titanium dioxide, it can be seen as a coating process. The substrate is generally coated glass, stainless steel, aluminum and the like; coating precursor can be directly used titanium dioxide powder may also contain other substances Chin.

Film growth by chemical vapor deposition (CVD) is one of the most important method of forming a thin film. It provides a relatively low temperature in a deposition method of a considerable amount of various elements and compounds. The principle is the use of volatile metal compound vapor to produce the desired compound by a chemical reaction, rapid cooling in a protective gas environment, is deposited on the substrate. The process consists of three stages: the material gasification, transport to the space near the substrate and is formed on the base coating layer. Usually gas-phase chemical reaction system activation methods are resistance wire heating, chemical flame heating, plasma heating, laser-induced, Y-ray radiation and other means. Precursor used in the reaction are of two kinds: Tiel4 and titanium alkoxide. The main operating conditions 
affect the film formation conditions include: the form of contact of the reactants, the reaction temperature, deposition time, concentration of reactants, and other carriers.

Sputtered film sputtering film's main principle is: the two parallel metal plates placed in Ar (low pressure environment, a pressure of about $40 \sim 250 \mathrm{~Pa}$ ), an anode, a cathode another piece of target material. Plus a DC voltage of several hundred volts, between the two plates to produce glow discharge, glow discharge between the plates of ions impinging on the cathode, the target material surface atoms will evaporate therefrom, and then deposited into forming a thin film on a substrate. Adjusting the discharge current, voltage and gas pressure, film formation can be achieved for each control factor.

\section{Conclusion}

Since the industrial revolution, scientific and technological progress of mankind in the economic development and the number on the scale has made remarkable achievements. However, excessive use of resources and energy, a lot of pollutant emissions, large-scale human-caused environmental disasters continue to occur, and some has become a global problem threatening human survival and development, and therefore, the effective use of resources and protection of the environment and degradation repair the environment is today's most important issue.

\section{References}

[1] V. Georgieva, M. Ristov, Electrodeposited cuprous oxide on indium tin oxide for solar applications, Solar Energy Materials \& Solar Cells, 2002, 73: 67-73.

[2] J.-N. Nian, C.-C. Hu, H. Teng, Electrodeposited p-type $\mathrm{Cu} 2 \mathrm{O}$ for $\mathrm{H} 2$ evolution from photoelectrolysis of water under visible light illumination, International Journal of Hydrogen Energy, 2008, 33: 2897-2903.

[3] W. Siripala, A. Ivanovskaya, T. F. Jaramillo, S.-H. Baeck, E. W. McFarland, A Cu2O/TiO2 heterojunction thin film cathode for photoelectrocatalysis, Solar Energy Materials and Solar Cells, 2003, 77(3): 229-237.

[4] T. D. Golden, M. G. Shumsky, Y. Zhou, R. A. VanderWerf, R. A. Van Leeuwen, J. A. Switzer, Electrochemical Deposition of Copper(I) Oxide Films, Chemistry of Materials, 1996, 8(10): 2499-2504.

[5] R. P. Wijesundera, M. Hidaka, K. Koga, M. Sakai, W. Siripala, Growth and characterisation of potentiostatically electrodeposited $\mathrm{Cu} 2 \mathrm{O}$ and $\mathrm{Cu}$ thin films, Thin Solid Films, 2006, 500(1-2): 241-246. 